\title{
Temporal Filtering of Nociceptive Information by Dynamic Activation of Endogenous Pain Modulatory Systems
}

\author{
Marc D. Yelle, Yoshitetsu Oshiro, Robert A. Kraft, and Robert C. Coghill \\ Department of Neurobiology and Anatomy and Division of Radiological Sciences, Wake Forest University School of Medicine, Winston-Salem, North \\ Carolina 27157-1010
}

\begin{abstract}
Endogenous pain control mechanisms have long been known to produce analgesia during "flight or fight" situations and to contribute to cognitively driven pain modulation, such as placebo analgesia. Afferent nociceptive information can also directly activate supraspinal descending modulatory systems, suggesting that these mechanisms may participate in feedback loops that dynamically alter the processing of nociceptive information. The functional significance of these feedback loops, however, remains unclear. The phenomenon of offset analgesia - disproportionately large decreases in pain ratings evoked by small decreases in stimulus intensity-suggests that dynamic activation of endogenous pain inhibition may contribute to the temporal filtering of nociceptive information. The neural mechanisms that mediate this phenomenon remain currently unknown. Using functional magnetic resonance imaging, we show that several regions of the midbrain and brainstem are differentially activated during offset analgesia. These activations are consistent with the location of areas such as the periaqueductal gray (PAG), rostral ventral medulla, and locus ceruleus that have substantial roles in descending inhibition of pain. This transient analgesia contributes to the temporal filtering of nociceptive information by producing a perceptual amplification of the magnitude and duration of decreases in noxious stimulus intensity. Together with the involvement of PAG and associated brainstem mechanisms in cognitively generated analgesia, the present observations suggest that the fundamental role of endogenous pain modulatory mechanisms is to dynamically shape the processing of nociceptive signals to best fit with the ever-changing demands of the environment.
\end{abstract}

\section{Introduction}

The midbrain and brainstem have long been known to contribute to top-down modulation of neuronal activity in spinal cord (Bernard, 1858; Sherrington and Sowton, 1915). One of the most crucial midbrain regions involved in descending control of pain, the periaqueductal gray (PAG), integrates afferent input from the spinal cord, cerebral cortex, as well as numerous brainstem nuclei (Beitz, 1982; Mantyh and Peschanski, 1982; Marchand and Hagino, 1983; Liu, 1986; Millan, 2002; Sillery et al., 2005). Efferent information from the PAG can then be transmitted directly to the spinal cord or can reach the spinal cord indirectly via brainstem regions such as the rostroventral medulla (Hamilton and Skultety, 1970; Mantyh and Peschanski, 1982; Mantyh, 1983a,b). Stimulation of the ventrolateral PAG results in antinociception and analgesia in animal and human studies (Mayer et al., 1971; Hosobuchi et al., 1977), whereas disruption of descending control systems is associated with chronic pain (Lautenbacher and Rollman, 1997; Staud et al., 2003a; Julien et al., 2005; Yarnitsky et al., 2008).

Received Sept. 28, 2008; revised April 2, 2009; accepted July 3, 2009.

This study was supported by National Institutes of Health Grants NS 39426 and DA20168. Special thanks to Dr. Paul Laurienti for his insightful suggestions regarding statistical analysis. We also thank the FMRIB (Functional Magnetic Resonance Imaging of the Brain) Image Analysis Group (Oxford University, Oxford, UK) for the FSL analysis software used in this study.

Correspondence should be addressed to Dr. Robert C. Coghill, Department of Neurobiology and Anatomy, Wake Forest University School of Medicine, Winston-Salem, NC 27157-1010. E-mail: rcoghill@wfubmc.edu. DOI:10.1523/JNEUROSCI.4648-08.2009

Copyright $\odot 2009$ Society for Neuroscience $\quad$ 0270-6474/09/2910264-08\$15.00/0
Spinal cord neurons in lamina I, V, as well as VI, VIII, and X project to the PAG (Swett et al., 1985; Pechura and Liu, 1986; Harmann et al., 1988), and, although these projections are relatively sparse, they appear to be functional. For example, peripheral nerve stimulation (PNS) can cause analgesia that may result from activation of descending noradrenergic pathways (Men and Matsui, 1994). Many of the PAG neurons that can be activated by PNS can also be activated by noxious cutaneous stimulation (Eickhoff et al., 1978). Together, this anatomic and physiologic evidence suggests that the spinal input to the PAG can create a dynamic inhibitory feedback circuit through a spinal-supraspinalspinal loop (Swett et al., 1985). Moreover, the ascending projections of the PAG also provide a substrate for a feedforward inhibition by targeting regions of the thalamus important in afferent nociceptive processing (Mantyh, 1983a). Both feedback and feedforward inhibition could contribute to the temporal and/or spatial filtering of nociceptive afferent information as it is relayed to higher sensory areas (Le Bars et al., 1979; Jones, 1992; Willis and Westlund, 1997). However, the involvement of endogenous inhibitory systems in the spatial and temporal filtering of nociceptive information remains poorly understood.

The phenomenon of offset analgesia provides a novel paradigm to investigate temporal filtering of nociceptive information and afferent signal-driven pain modulation (Grill and Coghill, 2002; Yelle et al., 2008; Derbyshire and Osborn, 2008). Offset analgesia is the disproportionately large decrease in perceived pain intensity evoked by slight decreases in stimulus intensity. This amplification of the perceptual representation of such small 
decreases in stimulus intensity is consistent with the action of a mechanism that produces dynamic temporal sharpening of nociceptive information (Yelle et al., 2008). To determine whether midbrain and brainstem regions known to be involved in the modulation of pain contribute to the temporal filtering of nociceptive information during offset analgesia, we examined supraspinal activity in human volunteers using functional magnetic resonance imaging (fMRI).

\section{Materials and Methods}

Subjects. All subjects (eight female, seven male) were right-handed, healthy, normal subjects between the ages of 21 and 34 years $(25.88 \pm$ 0.98 years, mean \pm SEM) and had no history of chronic pain or any neurological disorder and no detectable MRI contraindications. Subjects were asked not to take any analgesics within $48 \mathrm{~h}$ of the study. All female subjects reported using a reliable method of birth control and were not pregnant while participating in this study. The Institutional Review Board at Wake Forest University School of Medicine approved all procedures used in this experiment. Before participating in this study, every subject gave written, informed consent acknowledging that they (1) understood all methods and procedures used in the experiment, (2) would experience painful stimuli and undergo an MRI scan, and (3) were free to withdraw from the study at anytime.

Psychophysical training and assessment. Subjects reported to the laboratory for psychophysical training before their MRI session. Thermal stimuli were delivered via a $16 \times 16 \mathrm{~mm}$ Peltier device attached to the posterior aspect of the left lower leg (TSA-II; Medoc). Subjects were first presented with four series of eight thermal stimuli $\left(35^{\circ} \mathrm{C}, 43-49^{\circ} \mathrm{C}, 5 \mathrm{~s}\right)$ to familiarize them with using a trackball-controlled computerized visual analog scale (VAS) (verbal end points of "no pain sensation" and "most intense pain sensation imaginable") (Price et al., 1994; Koyama et al., 2004).

Subjects then were presented with four different series of thermal stimuli that were identical to those used for functional imaging. Each series consisted of four stimulus epochs: two $30 \mathrm{~s}$ constant $49^{\circ} \mathrm{C}$ stimuli and two three-temperature stimulus trains $\left(\mathrm{T} 1=49^{\circ} \mathrm{C}, \mathrm{T} 2=50^{\circ} \mathrm{C}\right.$, and $\mathrm{T} 3=$ $49^{\circ} \mathrm{C}$ ) (Fig. 1). Offset analgesia is evoked by the $1^{\circ} \mathrm{C}$ decrease from 50 to $49^{\circ} \mathrm{C}$ in the three-temperature stimulus train. The duration of each temperature in the three-temperature stimulus trains was varied to minimize the effects of expectation $(\mathrm{T} 1=6-9 \mathrm{~s}, \mathrm{~T} 2=7-8 \mathrm{~s}$, and $\mathrm{T} 3=13-17 \mathrm{~s})$. However, each stimulus train had a total duration of $30 \mathrm{~s}$. The order of each stimulus epoch within the thermal series as well as the order in which these series were presented to subjects was pseudorandomized to further minimize the effects of expectation. Additionally, the thermal probe was moved after each stimulus series to a completely distinct yet adjacent area of skin.

Structural and functional imaging. Subjects underwent functional imaging while being presented with noxious thermal stimulation on their left lower leg. The MRI session lasted $\sim 2 \mathrm{~h}$. Each MRI series consisted of four thermal stimuli in a classic block design. Each of the four experimental thermal series, described in psychophysical assessment and training section above, was presented to each subject twice: once it was rated on a computerized VAS and once without being rated. Subjects were instructed to lie in the scanner and were outfitted with MRI-compatible video display goggles, headphones, and a trackball. Subjects received cues through the headphones from the control room. During trials in which subjects were required to give pain ratings, subjects were instructed to open their eyes and provide real-time pain intensity ratings on the VAS projected in the goggles by moving the trackball. During unrated trials, subjects were asked to keep their eyes closed. Preliminary analyses indicated that pain-related activation was similar between rated and unrated trials, so these data were combined for all analyses.

Blood oxygenation level-dependent (BOLD) signal was used to assess regional brain activation. Functional data were acquired on a $1.5 \mathrm{~T}$ General Electric echo-speed Horizon LX scanner with a birdcage head coil (GE Medical Systems). Each fMRI series lasted 3 min and $12 \mathrm{~s}$ and consisted of 96 individual scans acquired using echo-planar imaging [echo
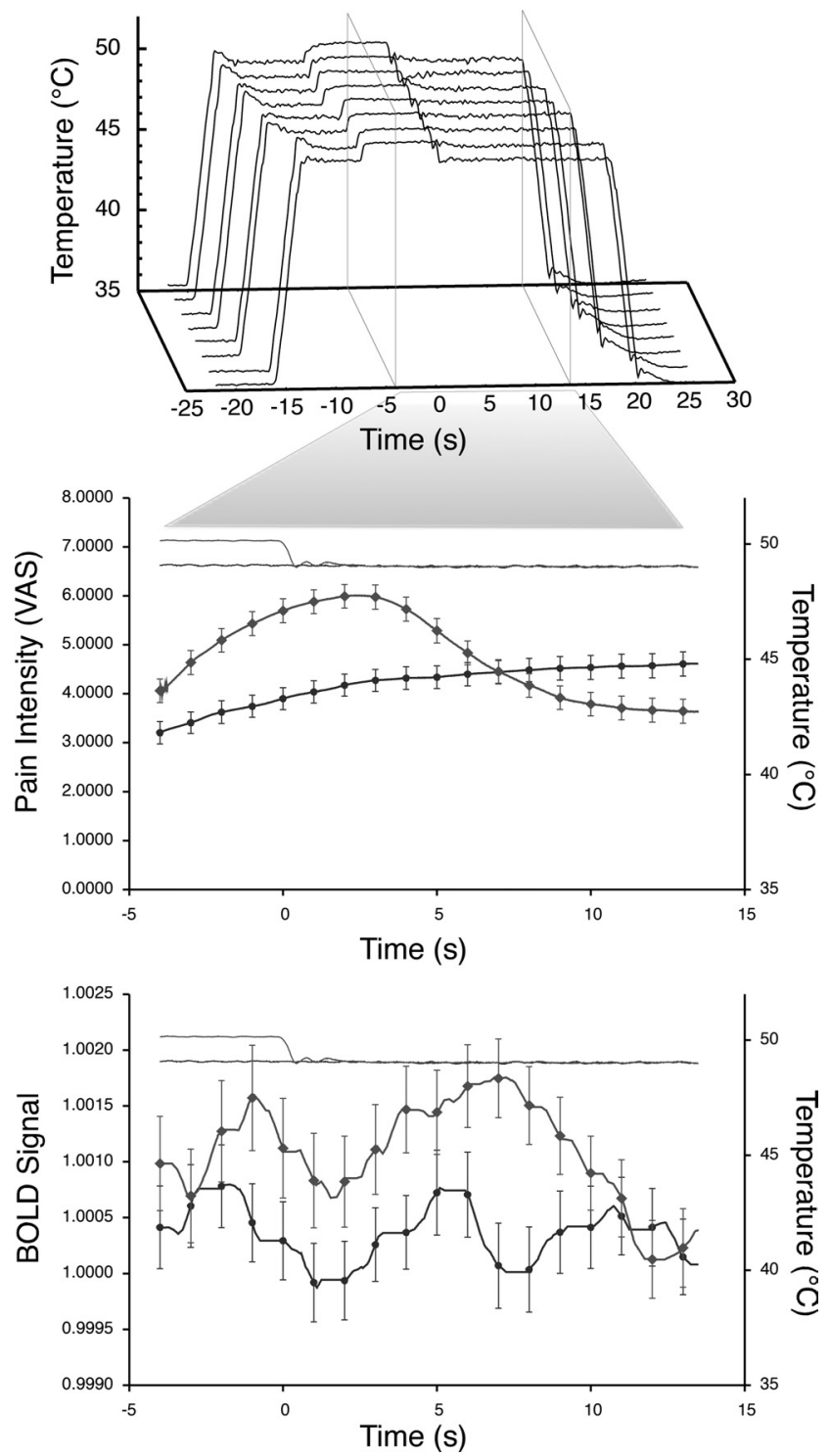

Figure 1. Time course of stimulus temperature, BOLD signal in the PAG, and pain intensity ratings. The $1^{\circ} \mathrm{C}$ temperature decrease in the three-temperature stimulus paradigm (top) was varied from $\sim 16$ to $20 \mathrm{~s}$ after the initiation of the stimulus train to minimize effects attributable to expectation. Because the durations of all three temperatures were varied, data from $4 \mathrm{~s}$ before the temperature decrease to $13.5 \mathrm{~s}$ after the temperature decrease represent the minimal time period at which all permutations of the stimulus train delivered equal temperatures (period between vertical planes). Accordingly, both pain intensity ratings (middle) and BOLD signal in the PAG (bottom) were averaged in this time window, with the time of the temperature decrease set to $0 \mathrm{~s}$. The $1^{\circ} \mathrm{C}$ temperature decrease evoked a $22.53 \%$ decrease in pain intensity ratings of the three-temperature stimulus (light gray diamonds) relative to the same time point during the constant $49^{\circ} \mathrm{C}$ stimulus (black circles). This decrease in pain ratings was associated with a statistically significant increase in the BOLD signal intensity of the PAG during the three-temperature stimulus (light gray diamonds) relative to that of the constant $49^{\circ} \mathrm{C}$ stimulus. BOLD signal was normalized to the first $20 \mathrm{~s}$ of the first rest period in each MRI series. All data displayed are derived from the mean \pm SEM response of all subjects.

time (TE), $40 \mathrm{~ms}$; repetition time (TR), $2 \mathrm{~s} ; 28 \times 5 \mathrm{~mm}$ thick slices; $3.72 \times$ $3.75 \mathrm{~mm}$ in-plane resolution; flip angle, $90^{\circ}$; no slice gap].

High-resolution structural scans were used to determine the location of functional changes in BOLD signal and, additionally, were used to generate a region of interest (ROI) of the periaqueductal gray. Structural scans were acquired using a three-dimensional (3-D) fast spoiled gradient recalled echo sequence with inversion preparation (inversion time, $600 \mathrm{~ms}$; TR, $9.1 \mathrm{~ms}$; flip angle, $20^{\circ}$; TE, $1.98 \mathrm{~ms}$; matrix, $256 \times 196 ; 124$ 
$1.5 \mathrm{~mm}$ slices; in-plane resolution, $0.9375 \times 0.9375 \mathrm{~mm}$; field of view, $24 \times 24 \mathrm{~cm})$.

Image processing. For image processing and statistical analysis, the functional image analysis package FSL [Center for Functional Magnetic Resonance Imaging of the Brain (FMRIB), University of Oxford, UK] was used. The functional data were motion corrected, spatially smoothed by a $5 \mathrm{~mm}$ full-width at half-maximum 3-D isotropic Gaussian kernel and temporally filtered (Gaussian-weighted least-squares straight line fitting, with $\sigma=37.5 \mathrm{~s}$ ). Intensity normalization was applied to each functional image to minimize effects of global signal fluctuations. The functional data were registered to their structural data using a sevenparameter linear 3-D transformation and then were transformed into standard stereotaxic space (Talairach and Tournoux, 1988) using a 12parameter linear 3-D transformation (Jenkinson and Smith, 2001).

Region of interest analysis. To test the hypothesis that activation of descending control systems occurs during offset analgesia, an ROI analysis was performed on the periaqueductal gray. This ROI was identified on the average structural image of all 15 subjects using anatomical landmarks with the reference of a detailed atlas and excluded the cerebral aqueduct (Duvernoy, 1995). A repeated measures ANOVA was used to determine whether activity within this region was significantly greater in the $8 \mathrm{~s}$ after the $1^{\circ} \mathrm{C}$ decrease from 50 to $49^{\circ} \mathrm{C}$ in the three-temperature stimulus train than during analogous time points in the constant temperature stimuli.

Assessment of regional brain activation during offset analgesia. To determine which brain areas outside of the PAG exhibited either increased or decreased activity during offset analgesia, all voxels within the brain were examined with a conventional general linear model-based statistical analysis. To accomplish this, two regressors [explanatory variables (EVs)] were constructed for each of the four thermal series. These two regressors (EVs) were designed to directly contrast offset analgesiarelated brain activation with pain-related brain activation. The offset analgesia regressor was set to -1 during rest periods and +1 during the $10 \mathrm{~s}$ after the decrease is stimulus intensity from $\mathrm{T} 2\left(50^{\circ} \mathrm{C}\right)$ to $\mathrm{T} 3\left(49^{\circ} \mathrm{C}\right)$. All other time points were set to zero (no interest). This regressor therefore contrasts offset analgesia-related activation with resting activation. The second regressor was designed to contrast pain-related activation (time matched to the offset analgesia regressor in the three-temperature stimulus epochs) with resting activation. Therefore, rest periods were set to -1 , and pain (during the same temporal window as the offset analgesia regressor) was set to +1 ; all other time points were set to 0 (no interest). Each model was convolved with a gamma function $(3 \mathrm{~s} \mathrm{SD}, 6 \mathrm{~s}$ lag) to better approximate the hemodynamic response.

Statistical analyses were performed using the improved linear model of FMRIB (Woolrich et al., 2001). For each individual, fixed effects general linear modeling (GLM) analyses were conducted using the regressors described above to identify brain activation associated with the modeled hemodynamic response function (Woolrich et al., 2001). Contrasts were constructed using these regressors to examine offset analgesia-related activation, pain-related activation, and differences between offset analgesia and pain. A Bayesian estimation method was then used for the higher-level random effects analyses to examine activation across subjects [FLAME (Beckmann et al., 2003; Woolrich et al., 2004)]. Regional changes in brain activation were then identified in $Z$ (Gaussianized T/F) statistic images that were thresholded using clusters determined by $Z>$ 2.3 and a (whole-brain corrected) cluster significance threshold of $p<$ 0.05 (Worsley et al., 1992).

Identification of brain activation correlated with that of the periaqueductal gray. To further characterize the relationship of regional changes in brain activation observed during offset analgesia with activation of the PAG, the time course of the BOLD signal within the PAG was used as a regressor in a GLM analysis. After statistical thresholding as above, this procedure identifies voxels exhibiting activation that is correlated with that of the PAG. Signal fluctuations associated with the white matter were identified and added to the statistical model as nuisance variables.

\section{Results}

\section{Psychophysics}

Subjects were instructed to provide real-time pain intensity ratings using a continuous VAS while undergoing an fMRI scan and receiving noxious thermal stimulation on their left lower leg. For the three-temperature stimulus trains, pain ratings were significantly lower after the decrease from $50^{\circ} \mathrm{C}$ (T2) to $49^{\circ} \mathrm{C}$ (T3) than pain ratings at the same time point for constant $49^{\circ} \mathrm{C}$ stimuli $\left(F_{(1,14)}=14.12 ; p<0.0021\right)$ (Fig. 1). This represents a $22.53 \pm$ $5.01 \%$ difference in pain ratings reported for a thermal stimulus of equal intensity and indicates that significant offset analgesia was evoked. This decrease in perceived pain intensity lasted from $\sim 7.5$ s before beginning to rise toward a level that would be evoked by a constant, 30 -s-duration, $49^{\circ} \mathrm{C}$ stimulus.

\section{Responses within the PAG region of interest}

When the time course of BOLD signal change within the periaqueductal gray region of interest was examined, signal intensity was found to dramatically increase after the decrease in stimulus intensity from $50^{\circ} \mathrm{C}$ (T2) to $49^{\circ} \mathrm{C}$ (T3) (Fig. 1). This increase in BOLD signal was significantly larger than the BOLD signal in the periaqueductal gray during constant noxious thermal stimulation $\left(49^{\circ} \mathrm{C}\right)$ at the same time point $\left(F_{(1,14)}=12.34 ; p<0.004\right)$. BOLD signal started to increase $\sim 1 \mathrm{~s}$ after the transition from $\mathrm{T} 2$ $\left(50^{\circ} \mathrm{C}\right)$ to $\mathrm{T} 3\left(49^{\circ}\right)$ and reached a peak $\sim 6 \mathrm{~s}$ after the $\mathrm{T} 2-\mathrm{T} 3$ transition. When the lag in hemodynamic response, generally between 4 and 7 s (Bandettini, 1999), is accounted for, the increase in the neural activity underlying the BOLD signal is initiated during the temperature shift and is maintained during the decreasing phase of reported pain intensity. Thus, the PAG exhibits activation during the period of offset analgesia.

\section{Subcortical activation associated with offset analgesia}

A hypothesis-independent whole-brain analysis revealed that the number of supraspinal loci exhibited a dynamic activation profile related to offset analgesia. Many of these areas were located in the brainstem and are associated with descending modulation of pain. During offset analgesia, a large, robust activation was identified in the midbrain at the level of the superior colliculus. This activation was strongest close to the cerebral aqueduct in a region that is consistent with the ventrolateral PAG (Fig. $2 \mathrm{~A}$ ). This same region did not demonstrate differential activation during constant noxious stimulation when compared with rest. Therefore, when directly contrasted, the PAG exhibited significantly greater activity during offset analgesia than during stimulation with noxious heat (Fig. 2A). The consistency between the region of interest findings and the activation detected in the PAG during the nondirected search suggests that the whole-brain analyses have sufficient sensitivity to detect focal activation at this level of the neuraxis.

Although the PAG has direct projections to the spinal cord, most of the modulatory efferent projections from the PAG reach to the spinal cord indirectly via multiple nuclei throughout the midbrain, brainstem, and cortical areas. Consistent with this anatomical organization, several other structures in the mesencephalon and brainstem were found to exhibit activity that was correlated with offset analgesia (Fig. 2). Although there are nuclei involved in descending control systems throughout the brainstem and pons, the vast majority of loci that are intrinsic to centrifugal pain modulatory circuits are located in the anterior limit of the pons and in the midbrain. Accordingly, activations within these regions have been identified when offset analgesia was con- 


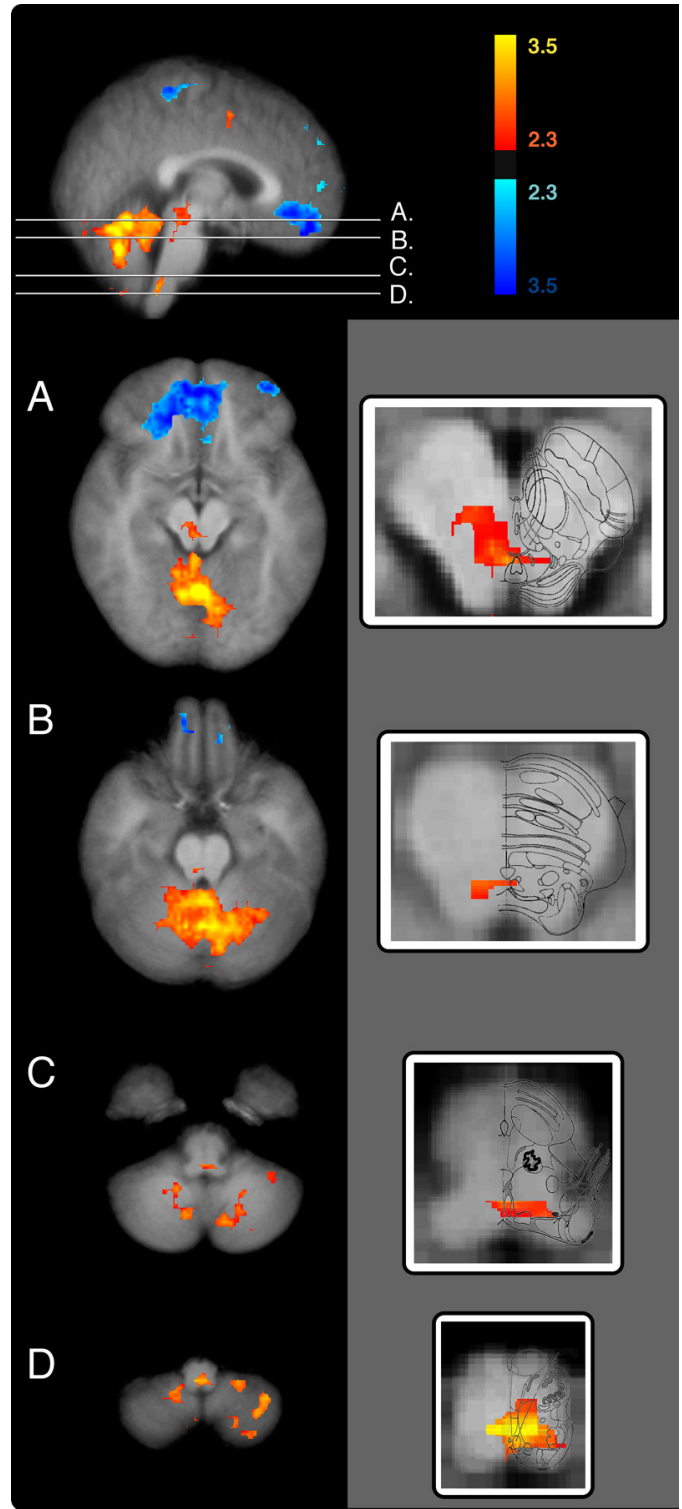

Figure 2. Regions of the midbrain and brainstem exhibiting significantly different activity during offset analgesia than during constant intensity $\left(49^{\circ} \mathrm{C}\right)$ stimulation. Regions exhibiting greater activity during offset analgesia (red-yellow) are consistent with the locations of the following: $\boldsymbol{A}$, the ventrolateral periaqueductal gray and dorsal raphe nucleus in the midbrain; $\boldsymbol{B}$, locus ceruleus (A6), the medial parabrachial nucleus (A7), the Kölliker-Fuse nucleus, and nucleus reticularis centralis superior ( $B 6$ and $B 8$ ) near the level of the superior cerebellar peduncle; $C$, the nucleus raphe magnus (B3), the nucleus reticularis gigantocellularis, and nucleus reticularis parvocellularis at the level of the pontine-medullary sulcus; and $\boldsymbol{D}$, an area consistent with the nucleus tractus solitarius in the inferior medulla. Atlas overlay adapted from Duvernoy (1995). In contrast to these regions, the ventromedial prefrontal cortex exhibited greater activity during constant intensity stimulation ( $\boldsymbol{A}$, blue). Image left corresponds to the subject's right. Colors correspond to z-score.

trasted with both rest and pain. In the midbrain, at the level of the superior colliculus, a large activation centered on the ventrolateral PAG extends ventrally to likely include the dorsal nucleus of raphe (B7) (Fig. 2A). At the level of the superior cerebellar peduncle, activation was identified in the caudal pons (Fig. $2 B$ ). This activation was centered on the midline and extended dorsolaterally. Activity in this region is consistent with the location of several important noradrenergic nuclei, including the nucleus ceruleus/locus ceruleus (A6), the medial parabrachial nucleus (A7), and Kölliker-Fuse nucleus, as well as the serotonergic cen- ter, nucleus reticularis centralis superior (B6 and B8). At the level of the ponto-medullary sulcus, activations were found in dorsal medial medulla (Fig. 2C). Several important serotonergic nuclei have been identified at this level of the neuraxis, including the nucleus raphe magnus (B3), the nucleus reticularis gigantocellularis, and nucleus reticularis parvocellularis. In the inferior medulla at the apex of the fourth ventricle, activation related to offset analgesia was identified (Fig. $2 D$ ). This activation in caudal medial medulla extended ventrally and laterally. These areas have been demonstrated previously to include noradrenergic nuclei $\mathrm{A} 1$ and A2, as well as the nucleus tractus solitarius.

Bilateral portions of the posterior thalamus were more active during offset analgesia than during rest (Fig. 3). Although the thalamus also exhibited greater activity during pain than during rest, activity was significantly greater during offset analgesia than during pain. This area of posterior thalamus is consistent with the pulvinar and ventral posterior lateral nucleus (VPL). In close proximity, activity at the posterior aspect of globus pallidus ipsilateral to stimulation was more active during offset analgesia than during pain, whereas the rostral globus pallidus was activated by both pain and offset analgesia (Fig. 3). Additionally, the cerebellum is frequently activated during functional imaging studies of pain (Coghill et al., 1999; Peyron et al., 2000) (Fig. 3). Although this study also found the cerebellum to be active during pain, there was significantly greater activity throughout most of the cerebellum during offset analgesia.

\section{Cerebral cortical changes during offset analgesia}

During pain, the contralateral primary somatosensory cortex (SI) was found to be more active than during rest, whereas the ipsilateral SI was deactivated (Fig. 3). During offset analgesia, both ipsilateral and contralateral SI were less active than they were at rest. This suggests that contralateral SI exhibits reduced nociceptive processing during offset analgesia.

Bilateral portions of the insular cortex were activated both during pain and offset analgesia (Fig. 3). However, a portion of contralateral insular cortex was more active during offset analgesia than during pain. The dorsolateral prefrontal cortex (DLPFC) was activated during noxious stimulation, and a similar region was activated during offset analgesia. However, when directly contrasted, a spatially restricted area of contralateral DLPFC was found to be more active during offset analgesia than during pain. The anterior cingulate cortex (ACC) was also found to be more active in each condition than rest, yet the mid-cingulate cortex (MCC) was more active during offset analgesia than during rest or painful stimulation (Fig. 3).

In addition to differential activation of cortical areas, two areas of the brain that are often reported to be deactivated during pain were found to be deactivated both during pain and offset analgesia. The precuneus was deactivated in both conditions but did not exhibit any significantly different deactivation when the two conditions were contrasted (Fig. 3). However, the ventral medial prefrontal cortex/subgenual anterior cingulate cortex was significantly more deactivated during offset analgesia than during pain (Fig. 3). Additionally, the dorsal medial prefrontal cortex is deactivated during offset analgesia (Fig. 3). This same region does not exhibit differential activation during painful stimulation.

\section{Correlation analysis of periaqueductal gray}

Numerous brain areas activated during offset analgesia also exhibited activity correlated with that of the PAG (Fig. 4). Subcortically, large portions of the cerebellum, thalamus, putamen, and 
caudate exhibited bilateral, yet asymmetric, activity that was correlated positively with that of the PAG. The amygdala and hippocampus also exhibited bilateral correlated activity, with more spatially extensive related activity occurring in the left hemisphere. At the cerebral cortical level, the ventromedial prefrontal cortex exhibited activity that was negatively related to that of the PAG.

\section{Discussion}

Endogenous pain modulatory mechanisms have long been known to powerfully influence the processing of nociceptive information. In general, their primary function has been viewed primarily as gain control, reducing the rostral flow of nociceptive information to facilitate escape, feeding, or mating in the presence of pain or to amplify nociceptive information during disease or injury (Millan, 2002; Fields, 2004). The present data indicate that the PAG and related brainstem regions important in pain inhibition are transiently activated during offset analgesia. With such dynamic activation, their capacity for gain control can be used for the temporal filtering of nociceptive information. Accordingly, activation of endogenous inhibitory systems during small decreases in noxious stimulus intensity produces analgesia and results in a disproportionately large perceptual change.

\section{The periaqueductal gray and} offset analgesia

Of all the supraspinal regions that exhibited activity correlated with offset analgesia, the activation within the PAG may be the most physiologically significant. The PAG was found to be more active during offset analgesia than during rest or during pain (Figs. 1-3). The activation was centered on the ventral PAG and extended laterally (Fig. 2). When this region of the PAG is electrically stimulated in patients with intractable pain, patients report analgesia and a feeling of "well-being" (Young, 1989). Electrical and pharmacological stimulation of the PAG can cause antinociception and analgesia in animals and humans, respectively (Mayer et al., 1971; Hosobuchi et al., 1977). The ventrolateral PAG receives both the afferent input and efferent output required for temporal filtering. Afferent input that drives neuronal activity within the PAG projects from the prefrontal cortex, amygdala, numerous medullary and pontine nuclei, including both serotonergic [nucleus raphe magnus (NRM), nucleus reticularis paragigantocellularis (NRPG), and nucleus tractus solitarius (NTS)], and noradrenergic [locus ceruleus (LC), parabrachial nucleus (PB), Kölliker-Fuse nucleus (KF)] nuclei, as well as sparse, yet functional spinal input (Beitz, 1982; Mantyh and Peschanski, 1982; Millan, 2002; Lovick and Bandler, 2005). The efferent connections of the PAG highlight its importance to centrifugal control of pain. The PAG projects directly to every level of the spinal cord (Castiglioni et al.,
1978; Mantyh, 1983b). Additionally, the PAG projects to other brainstem nuclei (NRM, NRPG, LC, PB, and NTS), to the thalamus, and to cortical regions involved in pain processing (Hamilton and Skultety, 1970; Beitz et al., 1983; Mantyh, 1983a,b; Jones, 1992; Lovick and Bandler, 2005; Sillery et al., 2005). Therefore, the ventrolateral PAG has both the afferent and efferent projections as well as the physiological capacity to cause antinociception and to significantly contribute to offset analgesia and temporal filtering.

\section{Role of brainstem inhibitory mechanisms in temporal filtering of nociceptive information}

Functional imaging of the brainstem can be complicated by technical factors, including $\mathrm{B}_{\Theta}$ local field inhomogeneity-induced signal loss, image distortion, poor spatial resolution, and motion artifacts (Tracey and Iannetti, 2006). Recently, several studies have successfully imaged the brainstem using optimized acquisition paradigms to minimize these technical challenges (Tracey et al., 2002; Topolovec et al., 2004; Dunckley et al., 2005). Although MRI acquisitions in the present investigation were performed 


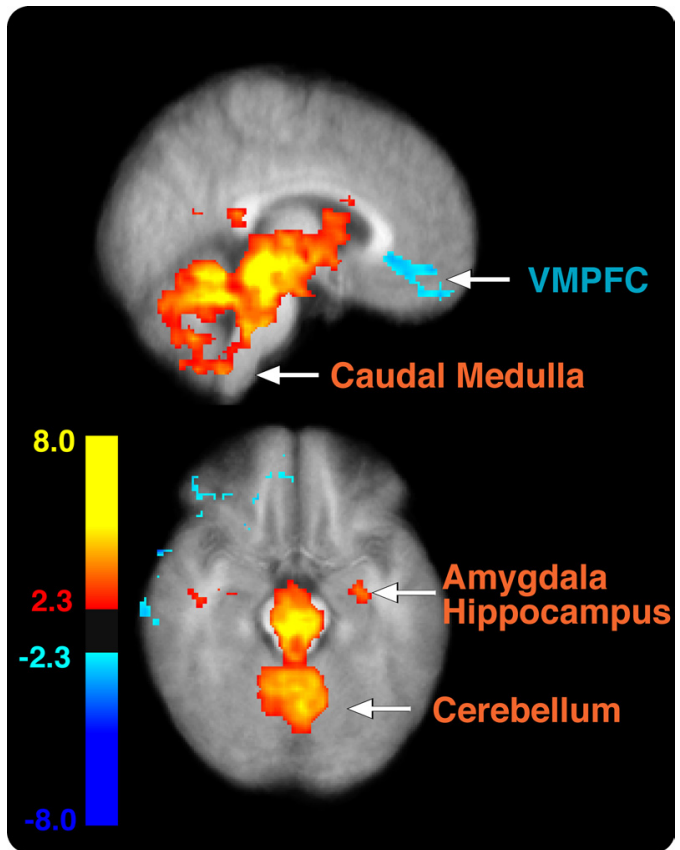

Figure 4. Brain regions correlated with the PAG. Numerous brain regions exhibited activity that was positively (red-yellow) or negatively (cyan-blue) related to that of the PAG. Image left corresponds to subject's right. Colors correspond to z-score.

conventionally, several lines of evidence suggest that the observed brainstem activations are related to offset analgesia. First, the activations observed in brainstem, as well as those in the midbrain, thalamus, and cortex, show BOLD activation in regions that are known to be involved in nociceptive processing. Second, the brainstem activations were highly focal and did not exhibit an edge-like spatial distribution characteristic of motion artifacts. Third, functional correlation of the PAG revealed focal and discrete sites of brainstem activity similar to those identified in the GLM analysis. Finally, the regressor used to identify these regions had a time course that was significantly different and not likely correlated with cardiac and respiratory cycles. Although we cannot conclusively identify each of the activated brainstem nuclei, areas of discrete activation were consistent with both serotonergic (NRM, dorsal raphe nucleus, lateral reticular nucleus, and NTS) and noradrenergic (LC, PB, KF, A1, and A5 cell groups) nuclei (Fig. 2) (Duvernoy, 1995). Each of these nuclei has very complex circuitry, with many reciprocal interconnections with other nuclei involved in pain modulation (Willis, 1988; Jones, 1992; Gebhart, 2004). Finally, each of these regions has been shown to produce analgesia when stimulated and has either direct or indirect output to the spinal cord (Willis, 1988; Jones, 1992; Millan, 2002; Gebhart, 2004) and therefore could potentially participate in the phenomenon of offset analgesia.

\section{Cerebellar responses during offset analgesia}

Extensive portions of the cerebellum were activated during offset analgesia and exhibited activity that was correlated with that of the PAG. Multiple regions of the cerebellum are commonly activated during pain (Borsook et al., 2008), and this activation may represent both pro-nociceptive and anti-nociceptive functions (Saab and Willis, 2003; Borsook et al., 2008). The robust activation of the cerebellum during offset analgesia is consistent with its putative role in the regulation of information flow to and from the somatosensory cortex (Borsook et al., 2008).

\section{Diencephalic and telencephalic responses during offset analgesia}

Several regions in the ventrobasal thalamus were activated during both offset analgesia and pain but exhibited significantly greater activity during offset analgesia than during pain (Fig. 3). The location of these activations is consistent with nuclei that are known to receive afferent nociceptive input. In chronic pain patients, stimulation of the VPL and ventral posteromedial nucleus results in analgesia (Turnbull et al., 1980), and, in primate studies, such stimulation has been shown to inhibit spinothalamic tract neurons (Gebhart et al., 1983). Inhibitory interconnections between neurons in the thalamus could account for these effects. Alternatively, these findings could reflect the activation of inhibitory interneurons in thalamus resulting from feedforward projections from PAG to thalamus (Mantyh, 1983a; Jones, 1992).

The contralateral primary somatosensory cortex was activated during pain when compared with rest, yet the ipsilateral SI was deactivated (Fig. 3). During offset analgesia, SI was deactivated when compared with pain and rest (Fig. 3). Although any reduction in ascending nociceptive input would be associated with decreased activity in SI, the finding of an SI deactivation during offset analgesia suggests that this region may be actively inhibited in a more direct manner during offset analgesia.

Regions such as the DLPFC, MCC, and insular cortex exhibited significantly greater activity during offset analgesia than during pain and, accordingly, could be involved in the generation or maintenance of offset analgesia. In humans, the DLPFC has connections with the PAG (Sillery et al., 2005; Hadjipavlou et al., 2006), and electrical stimulation of the prefrontal cortex can produce antinociception in animals (Hardy and Haigler, 1985). The MCC has frequently been reported to participate in pain processing and attention (Davis et al., 1997; Peyron et al., 2007). Additionally, the MCC may be involved in pain modulation. For example, the MCC exhibits increased activity during hypnotic suggestions for analgesia as well as opioid analgesia (Faymonville et al., 2000; Petrovic et al., 2002). Finally, the insular cortex receives input from the thalamus, amygdala, as well as SII (Friedman et al., 1986; Augustine, 1996). The insula is commonly thought to play a role in integrating sensory information with its emotional context (Coghill et al., 1994) and can be involved in pain modulation (Petrovic et al., 2002; Starr et al., 2009).

Other regions of the cortex were deactivated during offset analgesia. The precuneus and medial prefrontal cortex were deactivated during both pain and offset analgesia (Fig. 3). The medial prefrontal cortex and the precuneus have the highest resting metabolic rates in the brain (Raichle et al., 2001). These regions are commonly deactivated during sensory- and attention-related tasks. The dorsal medial prefrontal cortex (MPFC) was more deactivated during offset analgesia than during rest or pain (Fig. 3 ). The dorsal MPFC is suggested to contribute to introspectiveoriented mental activity (Gusnard et al., 2001). It is possible that subjects are engaged in rating stimulus intensity and that this deactivation is related to the subject's focused attention to the external stimulus.

\section{Expectations and offset analgesia}

Expectation and other cognitively driven factors may also contribute to offset analgesia. This study was specifically designed to minimize these potential confounds by varying the time of the T2-T3 temperature shift. Moreover, recent data indicate that, although the magnitude of offset analgesia increases over repeated testing sessions as expectations are learned, subjects still 
exhibit significant offset analgesia during the first testing session (Derbyshire and Osborn, 2008). Together, these findings suggest that offset analgesia and expectation represent independent phenomena that use common inhibitory mechanisms to modulate pain perception.

\section{Conclusion and implications}

Endogenous pain control mechanisms have long been known to modulate the processing of afferent sensory signals. The present data indicate that these mechanisms may contribute substantially to the temporal filtering of afferent information. Descending control mechanisms are commonly disrupted during chronic pain disorders (Lautenbacher and Rollman, 1997; Staud et al., 2003a; Julien et al., 2005; Yarnitsky et al., 2008). Consistent with this disruption, patients with several chronic pain disorders report painful aftersensations that may result from a loss of poststimulus inhibition (Noordenbos, 1959; Lindblom, 1985; Eide and Rabben, 1998; Gottrup et al., 2003; Staud et al., 2003b). Future experiments that investigate the temporal and spatial filtering of nociceptive information will shed considerable light on the mechanisms that support the sensory alterations that occur during chronic pain and could lead to novel treatments for chronic pain patients.

\section{References}

Augustine JR (1996) Circuitry and functional aspects of the insular lobe in primates including humans. Brain Res Brain Res Rev 22:229-244.

Bandettini PA (1999) Temporal resolution of functional MRI. In: Functional magnetic resonance imaging (Moonen PA, Bandettini PA, eds), pp 205-220. Berlin: Springer.

Beckmann CF, Jenkinson M, Smith SM (2003) General multilevel linear modeling for group analysis in FMRI. Neuroimage 20:1052-1063.

Beitz AJ (1982) The organization of afferent projections to the midbrain periaqueductal gray of the rat. Neuroscience 7:133-159.

Beitz AJ, Mullett MA, Weiner LL (1983) The periaqueductal gray projections to the rat spinal trigeminal, raphe magnus, gigantocellular pars alpha and paragigantocellular nuclei arise from separate neurons. Brain Res 288:307-314.

Bernard C (1858) Leçons sur la physiologie et la pathogie du système nerveux. Paris: Baillière.

Borsook D, Moulton EA, Tully S, Schmahmann JD, Becerra L (2008) Human cerebellar responses to brush and heat stimuli in healthy and neuropathic pain subjects. Cerebellum 7:252-272.

Castiglioni AJ, Gallaway MC, Coulter JD (1978) Spinal projections from the midbrain in monkey. J Comp Neurol 178:329-346.

Coghill RC, Talbot JD, Evans AC, Meyer E, Gjedde A, Bushnell MC, Duncan GH (1994) Distributed processing of pain and vibration in the human brain. J Neurosci 14:4095-4108.

Coghill RC, Sang CN, Maisog JM, Iadarola MJ (1999) Pain intensity processing within the human brain: a bilateral, distributed mechanism. J Neurophysiol 82:1934-1943.

Davis KD, Taylor SJ, Crawley AP, Wood ML, Mikulis DJ (1997) Functional MRI of pain- and attention-related activations in the human cingulate cortex. J Neurophysiol 77:3370-3380.

Derbyshire SW, Osborn J (2008) Enhancement of offset analgesia during sequential testing. Eur J Pain 12:980-989.

Dunckley P, Wise RG, Fairhurst M, Hobden P, Aziz Q, Chang L, Tracey I (2005) A comparison of visceral and somatic pain processing in the human brainstem using functional magnetic resonance imaging. J Neurosci 25:7333-7341.

Duvernoy H (1995) The human brainstem and cerebellum. New York: Springer.

Eickhoff R, Handwerker HO, McQueen DS, Schick E (1978) Noxious and tactile input to medial structures of midbrain and pons in the rat. Pain 5:99-113.

Eide PK, Rabben T (1998) Trigeminal neuropathic pain: pathophysiological mechanisms examined by quantitative assessment of abnormal pain and sensory perception. Neurosurgery 43:1103-1110.

Faymonville ME, Laureys S, Degueldre C, DelFiore G, Luxen A, Franck G,
Lamy M, Maquet P (2000) Neural mechanisms of antinociceptive effects of hypnosis. Anesthesiology 92:1257-1267.

Fields H (2004) State-dependent opioid control of pain. Nat Rev Neurosci 5:565-575.

Friedman DP, Murray EA, O’Neill JB, Mishkin M (1986) Cortical connections of the somatosensory fields of the lateral sulcus of macaques: evidence for a corticolimbic pathway for touch. J Comp Neurol 252:323-347.

Gebhart GF (2004) Descending modulation of pain. Neurosci Biobehav Rev 27:729-737.

Gebhart GF, Sandkühler J, Thalhammer JG, Zimmermann M (1983) Inhibition of spinal nociceptive information by stimulation in midbrain of the cat is blocked by lidocaine microinjected in nucleus raphe magnus and medullary reticular formation. J Neurophysiol 50:1446-1459.

Gottrup H, Kristensen AD, Bach FW, Jensen TS (2003) Aftersensations in experimental and clinical hypersensitivity. Pain 103:57-64.

Grill JD, Coghill RC (2002) Transient analgesia evoked by noxious stimulus offset. J Neurophysiol 87:2205-2208.

Gusnard DA, Akbudak E, Shulman GL, Raichle ME (2001) Medial prefrontal cortex and self-referential mental activity: relation to a default mode of brain function. Proc Natl Acad Sci U S A 98:4259-4264.

Hadjipavlou G, Dunckley P, Behrens TE, Tracey I (2006) Determining anatomical connectivities between cortical and brainstem pain processing regions in humans: a diffusion tensor imaging study in healthy controls. Pain 123:169-178.

Hamilton BL, Skultety FM (1970) Efferent connections of the periaqueductal gray matter in the cat. J Comp Neurol 139:105-114.

Hardy SG, Haigler HJ (1985) Prefrontal influences upon the midbrain: a possible route for pain modulation. Brain Res 339:285-293.

Harmann PA, Carlton SM, Willis WD (1988) Collaterals of spinothalamic tract cells to the periaqueductal gray: a fluorescent double-labeling study in the rat. Brain Res 441:87-97.

Hosobuchi Y, Adams JE, Linchitz R (1977) Pain relief by electrical stimulation of the central gray matter in humans and its reversal by naloxone. Science 197:183-186.

Jenkinson M, Smith S (2001) A global optimisation method for robust affine registration of brain images. Med Image Anal 5:143-156.

Jones SL (1992) Descending control of nociception. In: The initial processing of pain and its descending control: spinal and trigeninal systems (Light AR, ed), pp 203-277. New York: Kager.

Julien N, Goffaux P, Arsenault P, Marchand S (2005) Widespread pain in fibromyalgia is related to a deficit of endogenous pain inhibition. Pain 114:295-302.

Koyama Y, Koyama T, Kroncke AP, Coghill RC (2004) Effects of stimulus duration on heat induced pain: the relationship between real-time and post-stimulus pain ratings. Pain 107:256-266.

Lautenbacher S, Rollman GB (1997) Possible deficiencies of pain modulation in fibromyalgia. Clin J Pain 13:189-196.

Le Bars D, Dickenson AH, Besson JM (1979) Diffuse noxious inhibitory controls (DNIC). II. Lack of effect on non-convergent neurones, supraspinal involvement and theoretical implications. Pain 6:305-327.

Lindblom U (1985) Assessment of abnormal evoked pain in neurological pain patients and its relation to spontaneous pain: a descriptive and conceptual model with some analytical results. In: Advances in pain research and therapy (Fields HL, Dubner R, Cervero F, eds), pp 409-423. New York: Raven.

Liu RP (1986) Spinal neuronal collaterals to the intralaminar thalamic nuclei and periaqueductal gray. Brain Res 365:145-150.

Lovick T, Bandler R (2005) The organization of the midbrain periaqueductal grey and the integration of pain behaviours. In: The neurobiology of pain (Hunt SP, Koltzenburg M, eds), pp 267-287. New York: Oxford.

Mantyh PW (1983a) Connections of midbrain periaqueductal gray in the monkey. I. Ascending efferent projections. J Neurophysiol 49:567-581.

Mantyh PW (1983b) Connections of midbrain periaqueductal gray in the monkey. II. Descending efferent projections. J Neurophysiol 49:582-594.

Mantyh PW, Peschanski M (1982) Spinal projections from the periaqueductal grey and dorsal raphe in the rat, cat and monkey. Neuroscience 7:2769-2776

Marchand JE, Hagino N (1983) Afferents to the periaqueductal gray in the rat. A horseradish peroxidase study. Neuroscience 9:95-106.

Mayer DJ, Wolfle TL, Akil H, Carder B, Liebeskind JC (1971) Analgesia from electrical stimulation in the brainstem of the rat. Science 174:1351-1354. 
Men DS, Matsui Y (1994) Activation of descending noradrenergic system by peripheral nerve stimulation. Brain Res Bull 34:177-182.

Millan MJ (2002) Descending control of pain. Prog Neurobiol 66:355-474.

Noordenbos W (1959) Pain. Amsterdam: Elsevier.

Pechura CM, Liu RP (1986) Spinal neurons which project to the periaqueductal gray and the medullary reticular formation via axon collaterals: a double-label fluorescence study in the rat. Brain Res 374:357-361.

Petrovic P, Kalso E, Petersson KM, Ingvar M (2002) Placebo and opioid analgesia- imaging a shared neuronal network. Science 295:1737-1740.

Peyron R, Laurent B, García-Larrea L (2000) Functional imaging of brain responses to pain. A review and meta-analysis (2000). Neurophysiol Clin 30:263-288.

Peyron R, Faillenot I, Mertens P, Laurent B, Garcia-Larrea L (2007) Motor cortex stimulation in neuropathic pain. Correlations between analgesic effect and hemodynamic changes in the brain. A PET study. Neuroimage 34:310-321.

Price DD, Bush FM, Long S, Harkins SW (1994) A comparison of pain measurement characteristics of mechanical visual analogue and simple numerical rating scales. Pain 56:217-226.

Raichle ME, MacLeod AM, Snyder AZ, Powers WJ, Gusnard DA, Shulman GL (2001) A default mode of brain function. Proc Natl Acad Sci U S A 98:676-682.

Saab CY, Willis WD (2003) The cerebellum: organization, functions and its role in nociception. Brain Res Brain Res Rev 42:85-95.

Sherrington CS, Sowton SC (1915) Observations on reflex responses to single break-shocks. J Physiol 49:331-348.

Sillery E, Bittar RG, Robson MD, Behrens TE, Stein J, Aziz TZ, Johansen-Berg $\mathrm{H}$ (2005) Connectivity of the human periventricular-periaqueductal gray region. J Neurosurg 103:1030-1034.

Starr CJ, Sawaki L, Wittenberg GF, Burdette JH, Oshiro Y, Quevedo AS, Coghill RC (2009) Roles of the insular cortex in the modulation of pain: insights from brain lesions. J Neurosci 29:2684-2694.

Staud R, Robinson ME, Vierck CJ Jr, Price DD (2003a) Diffuse noxious inhibitory controls (DNIC) attenuate temporal summation of second pain in normal males but not in normal females or fibromyalgia patients. Pain 101:167-174.

Staud R, Cannon RC, Mauderli AP, Robinson ME, Price DD, Vierck CJ Jr (2003b) Temporal summation of pain from mechanical stimulation of muscle tissue in normal controls and subjects with fibromyalgia syndrome. Pain 102:87-95.

Swett JE, McMahon SB, Wall PD (1985) Long ascending projections to the midbrain from cells of lamina I and nucleus of the dorsolateral funiculus of the rat spinal cord. J Comp Neurol 238:401-416.

Talairach J, Tournoux P (1988) Co-planar sterotaxic atlas of the human brain. New York: Thieme.

Topolovec JC, Gati JS, Menon RS, Shoemaker JK, Cechetto DF (2004) Human cardiovascular and gustatory brainstem sites observed by functional magnetic resonance imaging. J Comp Neurol 471:446-461.

Tracey I, Iannetti GD (2006) Brainstem functional imaging in humans. Suppl Clin Neurophysiol 58:52-67.

Tracey I, Ploghaus A, Gati JS, Clare S, Smith S, Menon RS, Matthews PM (2002) Imaging attentional modulation of pain in the periaqueductal gray in humans. J Neurosci 22:2748-2752.

Turnbull IM, Shulman R, Woodhurst WB (1980) Thalamic stimulation for neuropathic pain. J Neurosurg 52:486-493.

Willis WD Jr (1988) Anatomy and physiology of descending control of nociceptive responses of dorsal horn neurons: comprehensive review. Prog Brain Res 77:1-29.

Willis WD, Westlund KN (1997) Neuroanatomy of the pain system and of the pathways that modulate pain. J Clin Neurophysiol 14:2-31.

Woolrich MW, Ripley BD, Brady M, Smith SM (2001) Temporal autocorrelation in univariate linear modeling of FMRI data. Neuroimage 14:1370-1386.

Woolrich MW, Behrens TE, Beckmann CF, Jenkinson M, Smith SM (2004) Multilevel linear modelling for FMRI group analysis using Bayesian inference. Neuroimage 21:1732-1747.

Worsley KJ, Evans AC, Marrett S, Neelin P (1992) A three-dimensional statistical analysis for CBF activation studies in human brain. J Cereb Blood Flow Metab 12:900-918.

Yarnitsky D, Crispel Y, Eisenberg E, Granovsky Y, Ben-Nun A, Sprecher E, Best LA, Granot M (2008) Prediction of chronic post-operative pain: pre-operative DNIC testing identifies patients at risk. Pain 138:22-28.

Yelle MD, Rogers JM, Coghill RC (2008) Offset analgesia: a temporal contrast mechanism for nociceptive information. Pain 134:174-186.

Young RF (1989) Brain and spinal stimulation: how and to whom! Clin Neurosurg 35:429-447. 